Leedy, M.G., \& Hart, B.L. (1985). Female and male sexual responses in female cats with ventromedial hypothalamic lesions. Behavioral Neuroscience, 99(5): 936-941. (Oct 1985) Published by the American Psychological Association (ISSN: 1939-0084)

\title{
Female and male sexual responses in female cats with ventromedial hypothalamic lesions
}

Mitzi G. Leedy and Benjamin L. Hart

\begin{abstract}
Following preoperative testing for receptivity, proceptivity, and male mating behavior, 27 female cats received either lesions in the anterior or posterior portion of the ventromedial hypothalamus $(\mathrm{VMH})$ or sham lesions. Neither of the $\mathrm{VMH}$ lesion placements reduced proceptivity and receptivity scores. However, the female mating pattern was significantly altered in that although females in both lesion groups initially allowed a stud male to mount, they usually did not allow the male to intromit. As in rodents, the $\mathrm{VMH}$ in the cat appears to be an important neural area for the display of normal female sexual behavior. Low levels of male sexual behavior were seen in the 3 groups preoperatively, and no changes were observed in the levels of male behavior following lesion placement. Thus, because lesions that disrupted female behavior did not affect male sexual behavior, it appears that the neural areas controlling homotypical and heterotypical sexual behaviors are not necessarily neurally linked.
\end{abstract}


Several lines of research point to the ventromedial hypothalamus (VMH) as being a primary neural area for the mediation of female sexual behavior. Estrogen-concentrating neurons have been found in the VHM in several vertebrate species, including the rat (Pfaff \& Keiner, 1973), hamster (Krieger, Morrell, \& Pfaff, 1976), and cat (Rees, Switz, \& Michael, 1980). Implantation of small amounts of estrogen into the VMH reliably results in receptivity in rats (Davis, McEwen, \& Pfaff, 1979) and guinea pigs (Morin \& Feder, 1974). Furthermore, lesions in the VMH result in decreased receptivity independently of effects of the lesions on gonadal hormone secretion in rats (Mathews \& Edwards, 1977a, 1977b) and hamsters (Malsbury, Kow, \& Pfaff, 1977). Knife cuts severing the lateral VMH connections have similar effects (Clark, Pfeifle, \& Edwards, 1981; Edwards \& Pfeifle, 1981; Malsbury, Strull, \& Daood, 1978). Reports on nonrodent species are less clear. In female sheep VMH lesions have been found to eliminate behavioral estrus while apparently not disrupting ovarian cycling (Clegg, Santolucito, Smith, \& Ganong, 1958). In a brief abstract, Sawyer and Robinson (1956) reported that lesions in the anterior, but not tuberal or posterior, hypothalamus eliminate female mating behavior in cats and that lesions of the mammillary region in rabbits were likewise effective in inhibiting copulation. No anatomical detail was given concerning the exact location and size of the lesions, nor were quantitative behavioral measures reported.

The display of heterotypical sexual behavior patterns has been reported for many mammalian species. Mounting, intromission, and ejaculatory behavior responses have been observed in female rats given long-term androgen treatment or electrical shock (Emery \& Sachs, 1975; Krieger \& Barfield, 1976). Female cats in natural estrus show many aspects of male mating behavior, such as taking a neck grip on another cat, mounting, and displaying pelvic thrusting while mounted (Prescott, 1970). These behaviors occur less frequently in females than in males, a result suggesting that although the females have the neural circuitry for male behaviors, it is probably held under neural inhibition.

It has been reported that $\mathrm{VMH}$ lesions that reduce female sexual behavior (lordosis) result in an enhancement of male sexual responding (Nance, Christensen, Shryne, \& Gorski, 1977). We had previously observed the converse of this effect (in male cats) that medial preoptic-anterior hypothalamic lesions, which impair male sexual behavior, potentiate female responding (Hart \& Leedy, 1983). Thus we were interested in determining whether VMH lesions in female cats would potentiate male sexual responding.

In the present study, we analyzed the effects of $\mathrm{VMH}$ lesions on mating activity in female cats, including measures of proceptive and receptive behaviors and measures of male sexual behavior. 


\section{METHOD}

\section{Subjects}

Female cats of mixed breed were obtained as adults from the campus Animal Resources Service. All animals were housed in colony pens of 6-9 cats, with individual perches provided for each cat. While receiving hormone injections, the cats were housed only with other cats receiving the same hormonal treatment.

\section{Surgical Procedures}

Anesthesia for the placement of $\mathrm{VMH}$ lesions was induced by ketamine $\mathrm{HCl}$ (Vetalar), and surgical anesthesia was maintained by the inhalant anesthetic fluothane (Halothane) and nitrous oxide. Heart rate, respiratory rate, and body temperature were monitored with a modified Beckman Vital Signs Monitor.

The stereotaxic procedure for making hypothalamic lesions has been reported in detail by Hart and Ladewig (1979) and Hart, Haugen, and Peterson (1973). Briefly, accurate electrode placement was determined by means of $X$-ray ventriculography, obtained by injecting a radiopaque contrast medium $(0.1 \mathrm{ml}$ of meglumine iothalamate, $60 \%)$ into the third ventricle. In this manner, the anterior commissure was easily visualized and was used as a reference. Inasmuch as the precise lesion site within the anterior portion of the hypothalamus had not been implicated in female cat mating behavior (Sawyer \& Robinson, 1956), the decision was made to place lesions in two distinct portions of the $\mathrm{VMH}$ : the anterior $\mathrm{VMH}(n=10)$ and posterior $\mathrm{VMH}$ $(n=9)$, an area comparable to the one that disrupts female sexual behavior in rodents. For both sites, bilateral electrode placements were used, 1.0-1.5 mm lateral to the midline and $5.0 \mathrm{~mm}$ ventral to the middle of the anterior commissure. The anterior $\mathrm{VMH}$ electrode placement was $2.5 \mathrm{~mm}$ posterior to the caudal edge of the anterior commissure, and the posterior placement was $1.0 \mathrm{~mm}$ more caudal. Radio-frequency lesions were made with a Kopf Model K13882 electrode with a thermistor tip, $0.7 \mathrm{~mm}$ in diameter and with an exposed length of $1.5 \mathrm{~mm}$. The lesions were made with a Kopf Model RFG-4 radio-frequency generator. Current was raised gradually until the tissue at the electrode tip was $67^{\circ} \mathrm{C}$, and the electrode was maintained at this temperature for $1 \mathrm{~min}$. Typically, such lesions are spherical and about $3 \mathrm{~mm}$ in diameter with these parameters. The procedures for sham operations were similar except that the electrode was lowered only to the level of the anterior commissure and no current was passed.

Following surgery, the animals were allowed to recover for 1 week in individual cages. A highly palatable food was available for the first 3 days postoperatively. By the end of 1 week, all animals were alert and eating normally. 


\section{Schedule of Testing and Operative Procedures}

All female cats were initially screened for willingness to mate. For these tests, they were given two injections of $0.2 \mathrm{mg}$ of estradiol cypionate (ECP) $48 \mathrm{hr}$ apart, and testing began $48 \mathrm{hr}$ after the second injection. The females were placed with a sexually active male cat on at least four occasions. Those that allowed the male to mate during these tests were then ovariohysterectomized and allowed to recover.

After approximately 3 weeks, androgen treatment was initiated. Subcutaneous injections of testosterone propionate (TP), at $5 \mathrm{mg} / \mathrm{kg}$ of body weight, were given every other day. This hormone injection regimen is effective in maintaining male sexual behavior in male cats (Hart \& Leedy, 1983). Preoperative testing for male sexual behavior was started following 2 weeks of TP treatment.

Three weeks after the last preoperative test for male sexual behavior, estrogen treatment was begun. Two injections of $0.2 \mathrm{mg}$ of ECP were given $48 \mathrm{hr}$ apart. Preoperative testing for female sexual behavior began $48 \mathrm{hr}$ after the second ECP injection. Two weeks after the last test for female behavior, all animals received either lesion placement or a control operation. Three more weeks passed before the subjects again received treatment with estrogen and the first postoperative series of tests for female sexual behavior. Two weeks with no hormones then elapsed before treatment with TP was initiated. Following 2 weeks of TP injections, the postoperative series of tests for male sexual behavior was given. Finally, 3 more weeks were allowed to elapse before a second postoperative estrogen treatment and series of female sexual behavior tests were given.

\section{Tests for Female Sexual Behavior}

Proceptivity was recorded in three tests in which subjects were placed in the testing chamber for 10 min with a sexually inactive, gonadally intact male cat that had sustained a medial preopticanterior hypothalamic brain lesion previously. This male was used because sexually active males usually mount females so rapidly that an observer has a difficult time obtaining a quantitative measure of proceptivity. Three measures were recorded: (a) approach of the male by the female in a nonagressive manner, (b) rubbing and rolling behavior while in the chamber, and (c) solicitation of a neck grip and mount by rubbing her neck and head against the male's face. A proceptivity score was determined by assigning one point for each of these three behaviors, for each test in which it occurred. Thus, for the series of three tests, the maximum proceptivity score was 9 .

In three separate tests for receptivity, given on different days than those for proceptivity, the female was placed in the test chamber for 15 min with a sexually vigorous male cat. At least two males were used during this series of tests. In these tests, we recorded the female's response to the male's mount or mount attempts as well as the number of times the female allowed the male to intromit (completed matings) in the $15 \mathrm{~min}$. A receptivity score was determined by assigning 1 point for each test in which each of the following behaviors occurred: (a) pelvic 
elevation—crouching with the forelimbs and elevating the pelvic region: (b) treading-alternate stepping movements of the hindlegs; and (c) tail deviation-movement of the tail to either side. The maximum receptivity score was 9 .

\section{Tests for Male Sexual Behavior}

In both the preoperative and postoperative periods, one series of five tests for male sexual behavior was given. In these tests, started after 2 weeks of TP treatment, the subject was placed in the testing chamber prior to the introduction of a stimulus female that was in behavioral estrus. Each test lasted 15 min during which time three measures of male sexual behavior were recorded: the occurrence of a neck grip in which the subject grapsed the stimulus female's neck scruff with her mouth, the occurrence of a properly oriented mount, and the display of pelvic thrusting while mounting. A male sexual behavior score was determined by assigning 1 point for each of these behaviors for each test in which it occurred. The maximum male score was thus 15 for the five tests.

\section{Response to Manual Stimulation}

Prior to each test for receptivity, all female cats were tested for the display of the receptive posture in response to manual stimulation of the anogenital region. While exerting mild pressure on the animal's lower back and holding the neck scruff, the experimenter stroked the perineal region. The occurrence of pelvic elevation, tail deviation and leg treading was recorded. The same scoring procedure was used as noted for receptivity, and the maximum score was 9.

\section{Postmortem Examination of the Brain}

Following the conclusion of behavioral testing, all subjects with brain lesions were anesthetized, and the brains were perfused with physiological saline followed by $10 \%$ formalin. The brains were blocked in the same plane used in the stereotaxic surgery and were stored in formalin. They were later sectioned at $40 \mu \mathrm{m}$ on a freezing microtome. Representative sections, at about $0.2 \mathrm{~mm}$ throughout the lesion area, were stained with cresyl violet and examined for location and extent of the lesions.

\section{RESULTS}

The results presented are taken only from those animals in which the $\mathrm{VMH}$ lesions were found to be in the designated areas. The data were discarded from 3 animals, which left 8 animals in each of the three groups. Figure 1 shows typical anterior and posterior $\mathrm{VMH}$ lesions at their maximum extent. The anterior lesions include the anterior hypothalamus caudal to the medial preoptic area and would presumably include the area damaged by Sawyer and Robinson 
(1956). The posterior lesions would be in a position comparable to those made in rodent species.
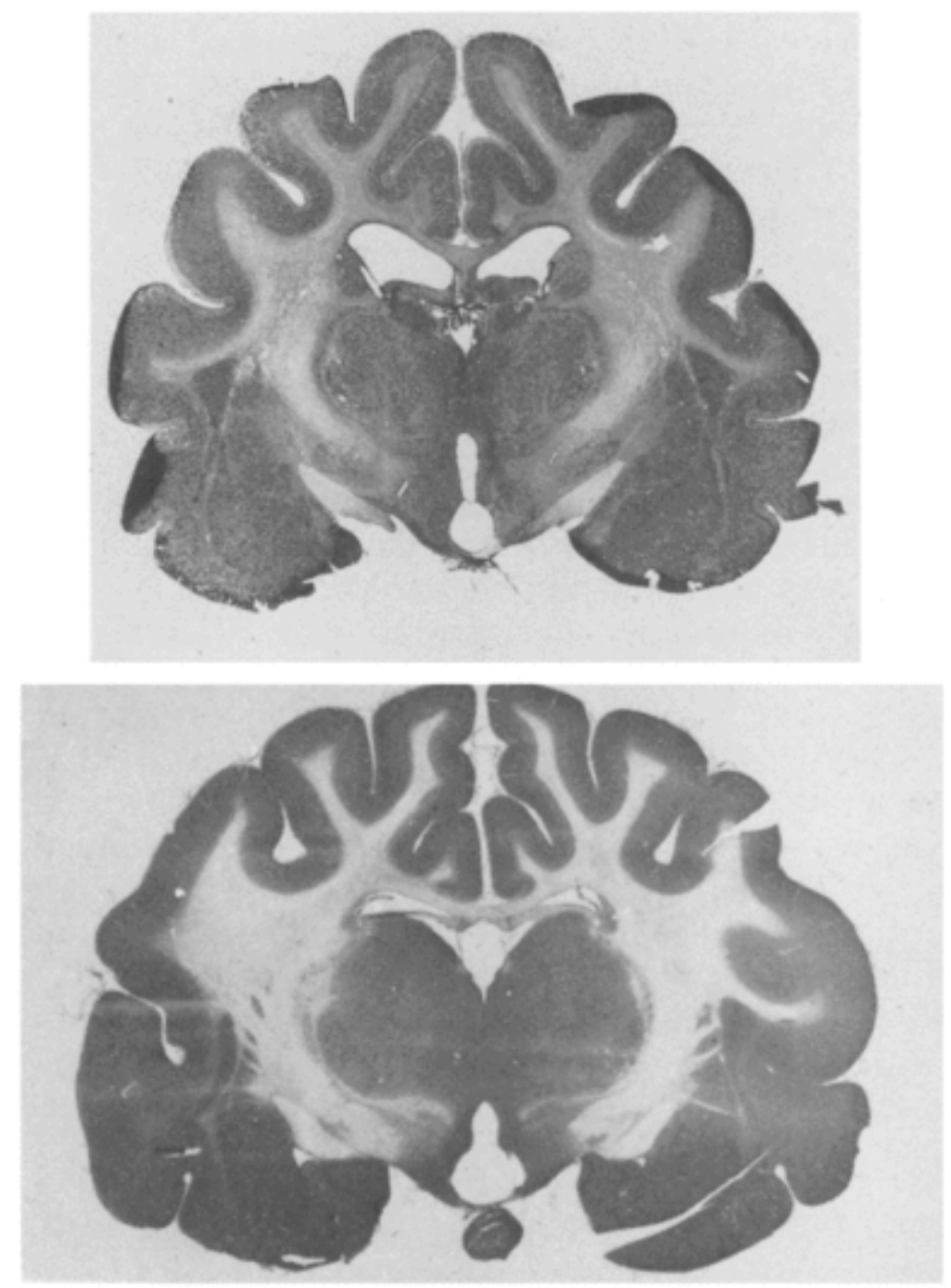

Figure 1. Photomicrograph of 40- $\mu m$ coronal sections, stained with cresyl violet, showing anterior (top) and posterior (bottom) ventromedial hypothalamic lesions. (See Plate A.)

\section{General Observations}

Although one might expect some irritability in cats with $\mathrm{VMH}$ lesions, no differences in manageability of the females with such lesions were noticed. Also, no weight gain was evident, as one might expect with $\mathrm{VMH}$ lesions. General observations made by laboratory personnel failed to reveal any behavioral changes in the subjects toward either their cagemates or the handlers. 
It was unexpected that those females with the anterior $\mathrm{VMH}$ lesions showed a marked degree of mammary hyperplasia postoperatively. None of the control animals and only 1 animal with the more posterior $\mathrm{VMH}$ lesion exhibited such hyperplasia. The condition was first noted approximately 2 weeks after the surgery and persisted for 2-3 weeks. No reoccurrence was observed in any of the animals.

\section{Female sexual behavior}

All animals showed high levels of female sexual behavior preoperatively, with no differences in receptivity or proceptivity scores between the groups (Figure 2). In postoperative tests, the females with either $\mathrm{VMH}$ lesion placement displayed typical proceptive behavior toward the sexually inactive males, and when placed with the sexually active males, they usually allowed them to mount and displayed pelvic elevation, leg treading, and tail deviation. Within a short period of time, usually less than $1 \mathrm{~min}$, the subjects would become aggressive, throw the males, and not allow any further mounts in that test period. The impairment in behavior was thus not represented in reduced proceptivity or receptivity scores. 


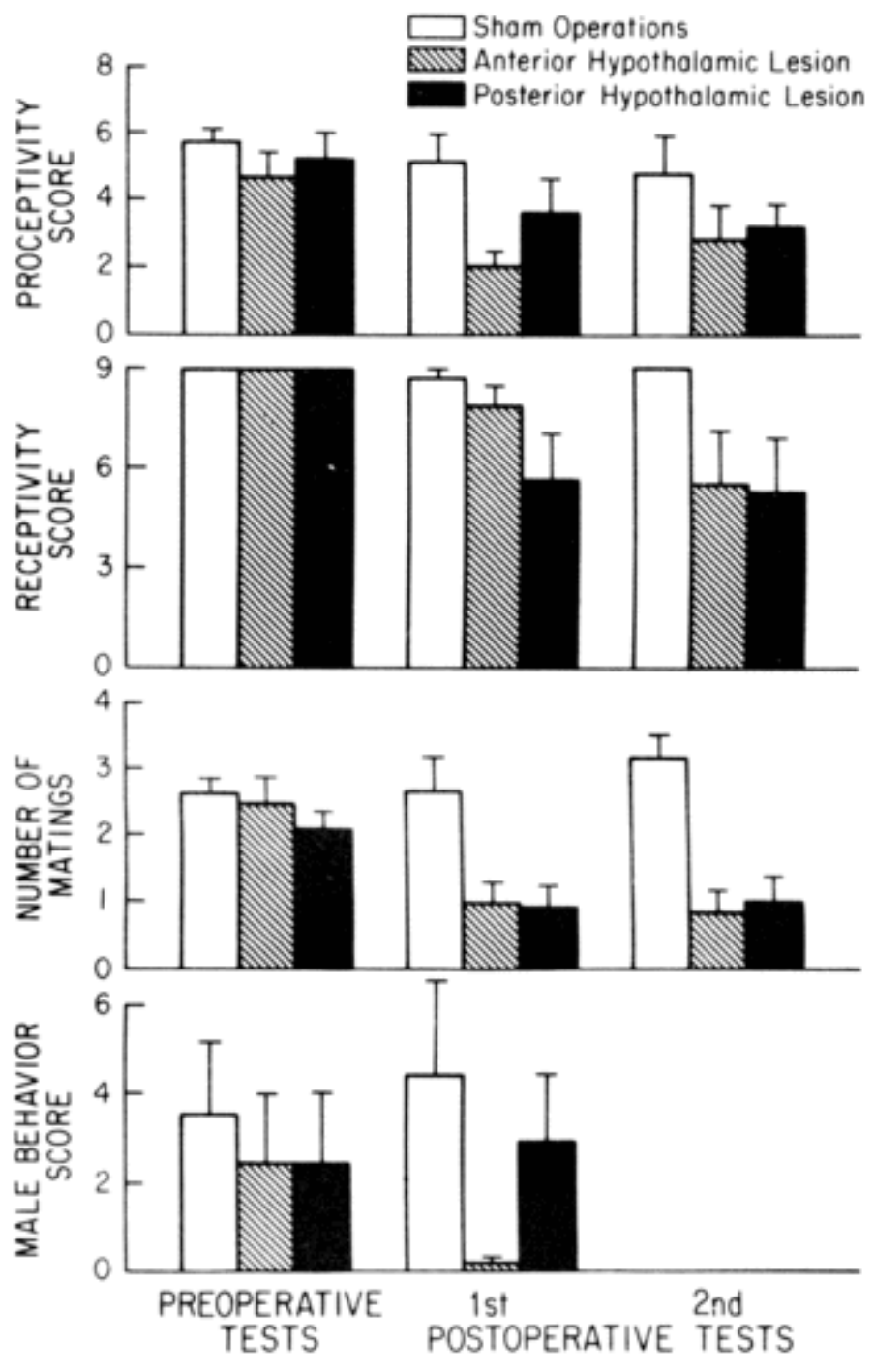

Figure 2. Mean ( \pm SE ) preoperative and first and second postoperative proceptivity scores, receptivity scores, and number of matings per test; and preoperative and postoperative male behavior scores for each group

Repeated measures analyses of variance were performed on the receptivity scores, proceptivity scores, and the number of matings completed in each test. Both the anterior $\mathrm{VMH}$ and the posterior $\mathrm{VMH}$ group showed lower levels of receptivity in the two postoperative series of tests, but the difference did not reach significance, $F(2,21)=2.81, p<.1$. Likewise, the two lesion groups showed moderately, but not significantly, lower proceptivity scores postoperatively, $F$ (2, $21)=2.27, p>$.1. However, the disruption in mating was evident when the number of completed matings in each test was considered. A significant difference between groups in the number of matings was found, $F(2,21)=7.2, p<.01$, as well as a significant Groups $\times$ Test 
Series interaction, $F(4,42)=6.3, p<.01$. Subsequent Scheffé's tests indicated that both the anterior $\mathrm{VMH}$ group and the posterior $\mathrm{VMH}$ group differed significantly from the control group on the postoperative tests, $F(2,21)=18.78, p<.01$ and $F(2,21)=17.82, p<.01$, respectively. The results of the female sexual behavior tests can be seen in Figure 2.

The scores for manual stimulation also revealed no change. The mean preoperative scores for anterior and posterior $\mathrm{VMH}$ groups were 7.88 and 6.62, respectively; the mean scores for the first preoperative series were 6.75 and 6.0 , respectively, and 7.0 and 6.5 for the second postoperative series, respectively.

\section{Male sexual behavior}

As Figure 2 shows, levels of male sexual behavior were low for all three groups of females for both the preoperative and postoperative tests. Only 8 of the 24 animals exhibited any male sexual behavior preoperatively. Analyses of the male behavior scores indicated no significant differences between the groups or across test series. The decrease in the male score seen in the anterior $\mathrm{VMH}$ group was due to the elimination of responding by the only 2 cats that displayed any male behavior preoperatively.

\section{DISCUSSION}

The results of the present study support earlier findings on rodents that the $\mathrm{VMH}$ is involved in the mediation of female sexual behavior (Clark et al., 1981; Edwards \& Pfeifle, 1981; Malsbury et al., 1977, 1978; Mathews \& Edwards, 1977a, 1977b). In the brief report by Sawyer and Robinson (1956), that anterior hypothalamic lesions impaired mating behavior in female cats, no mention was made of the nature of the impairment. The $\mathrm{VMH}$ lesions of the present study, although not eliminating female sexual responding, markedly reduced the number of completed matings. Quantitative measures of proceptivity and receptivity were not significantly decreased, but the VMH females were much less tolerant of the males' continued mounting behavior. This was probably not due to an increase in general aggressiveness, because the $\mathrm{VMH}$ females showed no more aggression toward a sexually inactive male than did the control females. The results are thus consistent with the general statement of Sawyer and Robinson that hypothalamic lesions impair mating behavior, although our experiment reveals that either anterior or posterior lesions in the $\mathrm{VMH}$ continuum will have this effect.

In the tests for female receptivity, the $\mathrm{VMH}$ animals appeared to be receptive during the initial portion of each mating test. They typically showed pelvic elevation, tail deviation, and treading of the hindlimbs in response to the male's mount. Also, these animals consistently showed these behaviors in response to manual stimulation. These behaviors are reflexive in nature, occurring in spinally transected female cats in response to manual stimulation following estrogen treatment (Hart, 1971). Although these behaviors are infrequently seen in nonspinal anestrous cats, presumably due to supraspinal inhibition, estrogen implanted in the posterior hypothalamus can induce them (Harris \& Michael, 1964). In a recent study, Rose and Smucker 
(1982) found that lesions in the central gray facilitated the receptive behavioral responses of pelvic deviation, tail deflection, and leg treading to manual stimulation in anestrous cats. They concluded that their lesions were effective by removing a neural area that, in the absence of estrogen, normally suppresses estrous behaviors. Neither the anterior nor the posterior $\mathrm{VMH}$ can be viewed as an estrogen-sensitive inhibitor of the central gray inhibitory area because the $\mathrm{VMH}$ lesions did not result in decreased receptive responses. If there are such estrogensensitive neurons, they may be located too diffusely to be eliminated by the electrode lesion technique.

In neither the cats of the present study nor rodents sustaining $\mathrm{VMH}$ lesions is the impairment of behavior as complete as reported for males following medial preopticanterior hypothalamic lesions. In some of the studies on female rodents, marked impairment, not elimination, of female responding was noted (Clark et al., 1981). Particularly noteworthy in this regard is that Pfaff and Sakuma (1979) found, as we did, that female rats sustaining VMH lesions responded to manual stimulation with the receptive posture.

The possibility that a brain area that is necessary for the display of homotypical sexual behavior patterns may also be involved in inhibiting the display of the opposite-sex behaviors was also explored in the present study because Nance et al. (1977) had found potentiation of male sexual behavior in female rats sustaining VMH lesions. Earlier findings of the converse of this effect in male cats are consistent with this concept, inasmuch as male cats with medial preopticanterior hypothalamic lesions, which eliminated male copulatory behavior, displayed increased female sexual responding (Hart \& Leedy, 1983). This concept was not supported by the present study, as male sexual behavior was not increased in either the anterior or the posterior VMH group. Our results are thus more along the lines of Mathews and Edwards (1977b) in finding no potentiation of male sexual behavior in female rats with VMH lesions than with those of Nance et al. (1977).

\section{REFERENCES}

Clark, A. S., Pfeifle, J. K., \& Edwards, D. A. (1981). Ventromedial hypothalamic damage and sexual proceptivity in female rats. Physiology and Behavior, 27, 597-602.

Clegg, M. T., Santolucito, J. A., Smith, J. D., \& Ganong, W. F. (1958). The effects of hypothalamic lesions on sexual behavior and estrous cycles in the ewe. Endocrinology, 62, 790-797.

Davis, P. G., McEwen, B. S., \& Pfaff, D. W. (1979). Localized behavioral implants in the ventromedial hypothalamus of female rats. Endocrinology, 104, 898-903.

Edwards, D. A., \& Pfeifle, J. K. (1981). Hypothalamic and midbrain control of sexual receptivity in the female rat. Physiology and Behavior, 26, 1061-1067.

Emery, D. E., \& Sachs, B. D. (1975). Ejaculatory pattern in female rats without androgen treatment. Science, 190, 484-486. 
Harris, G. W., \& Michael, R. P. (1964). The activation of sexual behavior by hypothalamic implants of oestrogen. Journal of Physiology, 171, 275-301.

Hart, B. L. (1971). Facilitation by estrogen of sexual reflexes in female cats. Physiology and Behavior, 7, 675-678.

Hart, B. L., Haugen, C., \& Peterson, D. M. (1973). Effects of medial preoptic-anterior hypothalamic lesions on mating behavior of male cats. Brain Research, 54, 177-191.

Hart, B. L., \& Ladewig, J. (1979). Effects of medial preoptic-anterior hypothalamic lesions on development of sociosexual behavior in dogs. Journal of Comparative and Physiological Psychology, 93, 566-573.

Hart, B. L., \& Leedy, M. G. (1983). Female sexual responses in male cats facilitated by olfactory bulbectomy and medial preoptic/anterior hypothalamic lesions. Behavioral Neuroscience, 97, 608-614.

Krieger, M. S., \& Barfield, R. J. (1976). Masculine sexual behavior: Pacing and ejaculatory patterns in female rats induced by electrical shock. Physiology and Behavior, 16, 671-675.

Krieger, M. S., Morrell, J. I., \& Pfaff, D. W. (1976). Autoradiographic localization of estradiolconcentrating cells in female hamster brain. Neuroendocrinology, 22, 193-205.

Malsbury, C. W., Kow, L. M., \& Pfaff, D. W. (1977). Effects of medial hypothalamic lesions on the lordosis response and other behaviors in female golden hamsters. Physiology and Behavior, 19, 223-237.

Malsbury, C. W., Strull, D., \& Daood, J. (1978). Half cylinder knife cuts anterolateral to the ventromedial nucleus reduce sexual receptivity in female golden hamsters. Physiology and Behavior, 21, 79-87.

Mathews, D., \& Edwards, D. A. (1977a). Involvement of the ventromedial and anterior hypothalamic nuclei in the hormonal induction of receptivity in the female rat. Physiology and Behavior, 19, 319-326.

Mathews, D., \& Edwards, D. A. (1977b). The ventromedial nucleus of the hypothalamus and the hormonal arousal of sexual behavior in the female rat. Hormones and Behavior, 8, 40-51.

Morin, L. P., \& Feder, H. H. (1974). Intracranial estradiol benzoate implants and lordosis behavior of ovariectomized guinea pigs. Brain Research, 70, 95-102.

Nance, D. M., Christensen, L. W., Shryne, J. E., \& Gorski, R. A. (1977). Modifications in gonadotropic control and reproductive behavior in the female rat by hypothalamic and preoptic lesions. Brain Research Bulletin, 2, 307-312.

Pfaff, D. W., \& Keiner, M. (1973). Atlas of estradiol-concentrating cells in the central nervous system of the female rat. Journal of Comparative Neurology, 151, 121-158. 
Pfaff, D. W., \& Sakuma, Y. (1979). Deficit in the lordosis reflex of female rats caused by lesions in the ventromedial nucleus of the hypothalamus. Journal of Physiology, 288, 203-210.

Prescott, R. G. W. (1970). Mounting behavior in the female cat. Nature, 228, 1106-1107.

Rees, H. D., Switz, G. J., \& Michael, R. P. (1980). The estrogen-sensitive neural system in the brain of female cats. Journal of Comparative Neurology, 193, 789-804.

Rose, J. D., \& Smucker, L. K. (1982). Central gray lesions produce estrous behavioral responses in hormonally anestrous cats. Behavioral and Neural Biology, 35, 84-90.

Sawyer, C. H., \& Robinson, B. (1956). Separate hypothalamic areas controlling pituitary gonadotropic function and mating behavior in female cats and rabbits. Endocrine Society Abstracts, 16, 914-915. 